\title{
Design of Flight Controllers based on Simplified LPV model of a UAV
}

\author{
Kannan Natesan, Da-Wei Gu, Ian Postlethwaite and Jianchi Chen
}

\begin{abstract}
In this paper, two strategies for the design of controllers based on a simplified LPV model of a UAV (longitudinal flight dynamics) are presented. The simplified LPV model is first derived from a UAV LPV model over the entire range of the cruise speed. The dependence of the LPV model on the varying parameter is reformulated in terms of a $\mu$ synthesis problem. A straight $\mu$ design and a gain-scheduling $\mu$ control scheme have been considered. Simulation results of the closed loop system comprising the controllers and original state-space models are presented and compared.
\end{abstract}

\section{INTRODUCTION}

$\mathrm{G}$ ain scheduling is an important and intrinsic part of any flight controller design process. While the classical gain-scheduling techniques use a family of equilibrium operating points for obtaining the corresponding controllers, the alternative continuous gain scheduling approach has gained increasing attention in recent years. This approach directly exploits the dependence of the linear state-space models on the scheduling parameter. Such systems known as Linear Parameter-Varying (LPV) systems can be expressed as:

$$
\begin{aligned}
& \dot{x}=A(\theta(t)) x+B(\theta(t)) u \\
& y=C(\theta(t)) x+D(\theta(t)) u
\end{aligned}
$$

where $\theta(\mathrm{t})$ is the varying parameter [1]. The main aim in the control of LPV systems is to guarantee closed-loop stability and performance for all possible varying parameters. In $[2,3]$, the scaled small gain theorem is used for the design of controllers for LPV systems that can be expressed in LFT form. While [2,3] use a modification of the small gain theorem to prove stability, performance in the sense of $\mathrm{L}_{2}$ norm is guaranteed in $[4,5]$ by obtaining a single quadratic Lyapunov function for all possible variations of the plant. It is however assumed that the parameters enter the LPV model in an affine fashion. In [6], the derivation technique is extended using the bounded real lemma formulation of $\mathrm{H}_{\infty}$ performance. The controller is then obtained by solving a system of Linear Matrix Inequalities (LMIs). Again the parameter dependence is assumed to be affine and the timevarying parameter $\theta$ is assumed to vary over a polytope of vertices. Recent approaches to controller synthesis for LPV systems include the use of unstructured scaling matrices at different vertices of the parameter region [7] and quadratic

Manuscript received March 8, 2006. This research work is supported by the BAE Systems and UK Engineering and Physical Sciences Research Council.

The authors are with the Department of Engineering, University of Leicester, LE1 7RH, UK. Phone: +44-116-2560; fax: +44-1162522619; e-mail: dag@leicester.ac.uk.
LFT Lyapunov functions and full-block multipliers [8].

While the satisfaction of robust stability and performance for LPV systems is the ultimate goal in controller synthesis, the modeling of LPV systems in itself is an important task. Specifically, the dynamics of aircraft and missiles are complex functions of various parameters including total velocity, height, angle of attack and sideslip angle. The most common method of obtaining an LPV model is through the Jacobian linearization of nonlinear dynamics at different operating points throughout the flight envelope [9, 10]. Other methods include state transformation and function substitution (the reader is referred to [10] for a detailed comparison of different LPV modelling techniques). The simpler the LPV model, the more tractable the problems of controller design and closed loop analysis are. In this paper, an LPV model of a UAV (Uninhabited Air Vehicle) is obtained through a series of approximations of the elements of the state-space matrices based on the understanding of flight dynamics, which thus simplifies the controller design process. Section 2 presents the LPV modelling of the UAV. The simplified LPV model is then used in Section 3 and 4 to obtain controllers through $\mu$-synthesis approaches. The first approach is to obtain a single $\mu$ controller to satisfy stability and performance specifications over the entire operating envelope and the second approach is to obtain a gainscheduling $\mu$ control scheme that is a function of the varying parameter. Section 4 also presents a comparative analysis of both the control schemes and Section 5 concludes the paper.

\section{LPV MODELLING OF UAV}

The aircraft considered in this paper is a generic model representative of the class of UAV's that operate at low Reynolds number. The linear longitudinal models of the UAV are obtained from the local linearisations of the 6degree of freedom nonlinear dynamic model and represented as:

$$
\left[\begin{array}{c}
\dot{u} \\
\dot{w} \\
\dot{\theta} \\
\dot{q}
\end{array}\right]=\left[\begin{array}{cccc}
X_{u}(V) & X_{w}(V) & -g \cos \left(\theta_{1}(V)\right. & X_{q}(V) \\
Z_{u}(V) & Z_{w}(V) & Z_{\theta}(V) & Z_{q}(V) \\
0 & 0 & 0 & \mathbf{1} \\
M_{u}(V) & M_{w}(V) & M_{\theta}(V) & \boldsymbol{M}_{\boldsymbol{q}}(V)
\end{array}\right]\left[\begin{array}{c}
u \\
w \\
\theta \\
q
\end{array}\right]+\left[\begin{array}{cc}
\boldsymbol{X}_{\delta \boldsymbol{e}}(V) & \boldsymbol{X}_{\delta \boldsymbol{t}}(V) \\
\boldsymbol{Z}_{\delta \boldsymbol{e}}(V) & \boldsymbol{Z}_{\delta \boldsymbol{t}}(V) \\
0 & \boldsymbol{0} \\
\boldsymbol{M}_{\delta \boldsymbol{e}}(V) & \boldsymbol{M}_{\delta \boldsymbol{t}}(V)
\end{array}\right]\left[\begin{array}{l}
\delta_{e} \\
\delta_{t}
\end{array}\right]
$$

The perturbed states in Eq. (2) are velocity $(u)$ along the $\mathrm{X}$-axis of the body-axes coordinate system, velocity $(w)$ along the z-axis of the body-axes coordinate system, pitch attitude $(\theta)$ and pitch rate $(q) . \delta_{\mathrm{e}}$ is the elevator input and $\delta_{\mathrm{t}}$ is the throttle input. The stability and control derivatives in Eq. (2) are functions of total velocity and height. However, 
for UAVs the variation in air density due to change in height is negligible and therefore the only varying parameter is the total velocity $\mathrm{V}$ that varies from $22 \mathrm{~m} / \mathrm{sec}$ to $72 \mathrm{~m} / \mathrm{sec}$. The dependence of the dimensional derivatives in Eq. (2) on velocity is found by using the method of least-squares curve fit. While the derivatives vary in both linear and quadratic fashions as functions of velocity, only the linear dependence is used to make the problem simple and tractable. The coefficients that most influence the dynamics of the UAV are found by fixing all coefficients in Eq. (2) at their average values, while in turn varying each coefficient over its entire range. The frequency and time responses of the model are then analyzed at various trim velocities to determine how a particular coefficient influences the dynamic characteristics. Proceeding in this way, it is determined that the coefficients $\mathrm{X}_{\mathrm{q}}, \mathrm{Z}_{\theta}, \mathrm{Z}_{\mathrm{q}}, \mathrm{M}_{\mathrm{u}}, \mathrm{Z}_{\delta \mathrm{e}}$ and $\mathrm{M}_{\delta \mathrm{e}}$ are the most significant ones in the sense that any variation in these coefficients would introduce large changes in the model response. This is expected, since any variation in $X_{q}$ and $Z_{\theta}$ mainly affects the phugoid damping and variation in $Z_{q}$ affects the damping and frequency of the phugoid mode. Variation in $\mathrm{M}_{\mathrm{u}}$ affects primarily the short period frequency and variations in $Z_{\delta \mathrm{e}}$ and $\mathrm{M}_{\delta \mathrm{e}}$ mainly affect the amplitude of the dynamic response of the aircraft.

Since the derivation of the simple LPV model involves numerous approximations and assumptions, it is worthwhile to compare the actual plant model and the derived LPV model. For this purpose, longitudinal models at 3 representative flight speeds are considered. Figures 1, 2 and 3 show the frequency responses of the actual plant model and the LPV model at 22, 47 and $72 \mathrm{~m} / \mathrm{sec}$, where the measured outputs are the total velocity, height and pitch rate. The frequency responses at 22 and $72 \mathrm{~m} / \mathrm{sec}$ show marked difference around the phugoid frequency and short-period frequency while the difference is negligible at the flight speed of $47 \mathrm{~m} / \mathrm{sec}$. Any variation in the dynamic characteristics can be treated as uncertainties against which the controller should be robust.

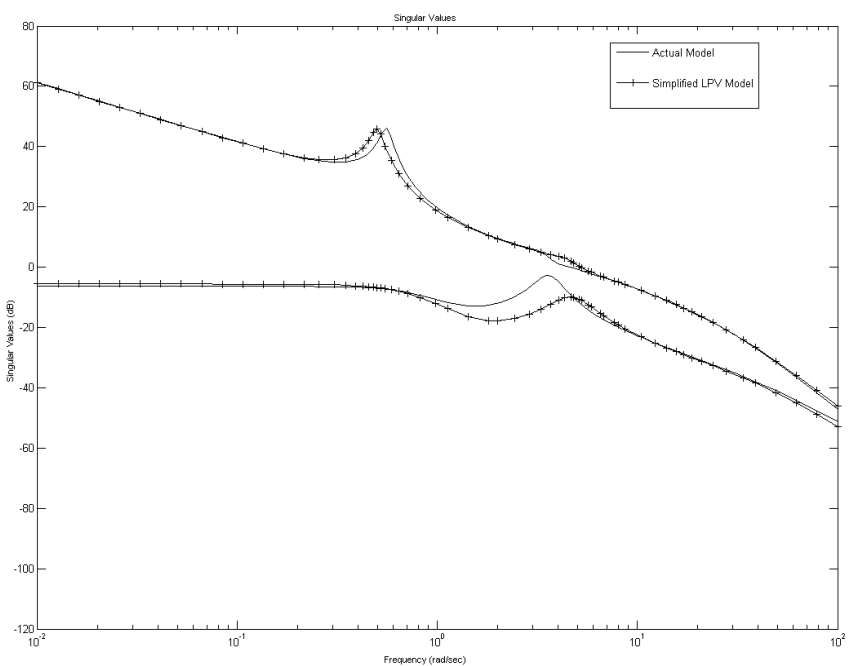

Fig. 1. Frequency Responses of the Actual Model and Simple LPV Model at $22 \mathrm{~m} / \mathrm{sec}$

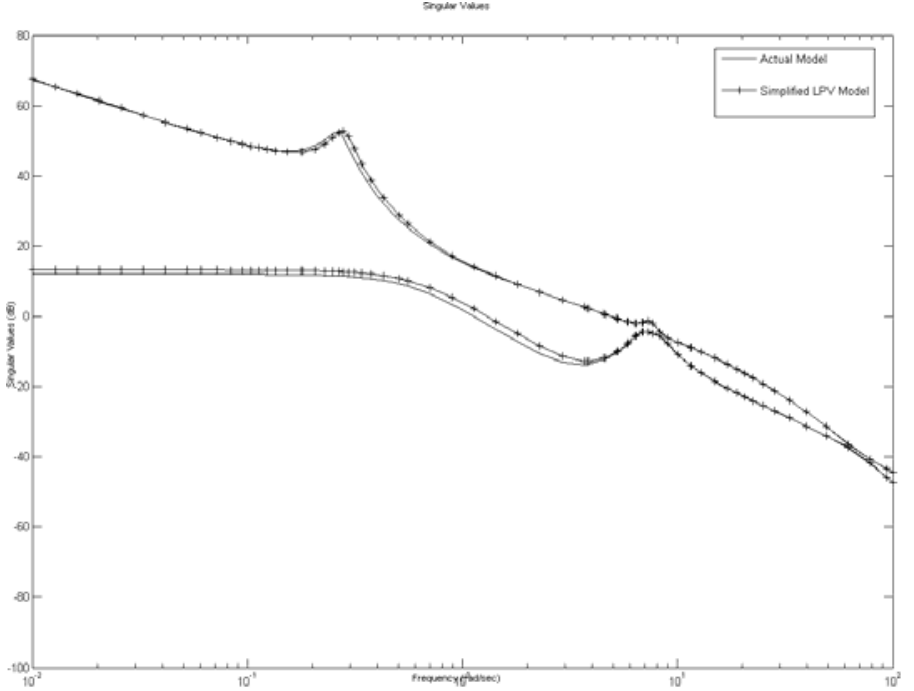

Fig. 2 Frequency Responses of Actual Model and LPV Model at 47 $\mathrm{m} / \mathrm{sec}$

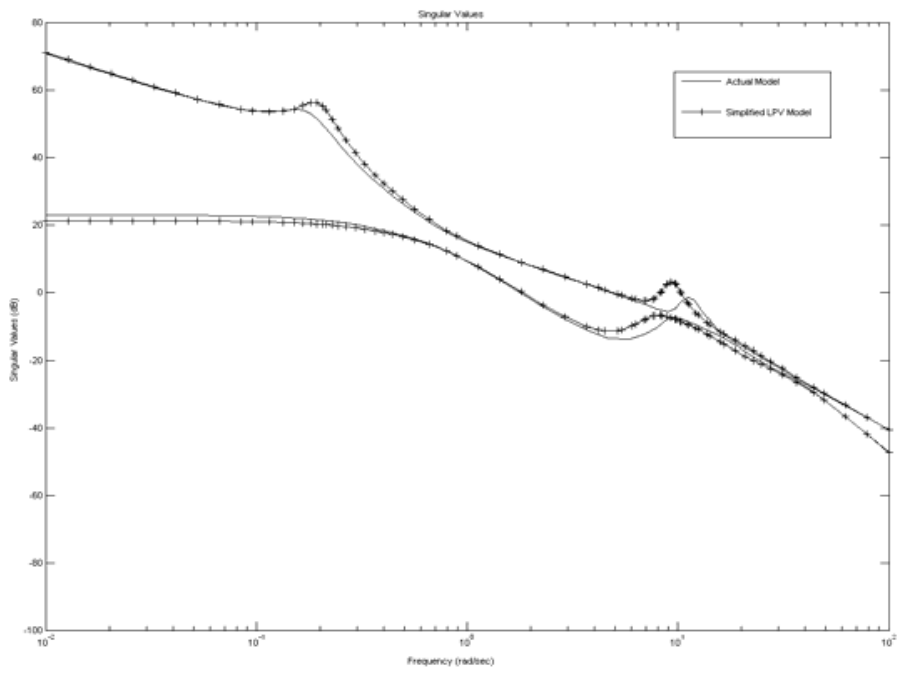

Fig. 3 Frequency Responses of Actual Model and LPV Model at 72 $\mathrm{m} / \mathrm{sec}$

III. DESIGN OF CONTROLLERS USING $\mu$ SYNTHESIS

Fig. 4 shows the closed loop interconnection for controller synthesis.

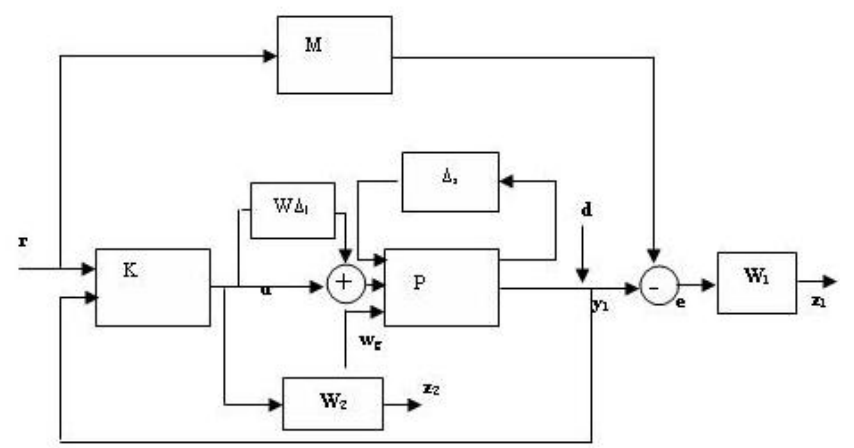

Fig. 4. Closed Loop Interconnection for Controller Synthesis $\mathrm{P}$ is the augmented nominal model obtained for the 
varying parameter $\mathrm{V}=0$ in the LPV model in Eq. (2). Sensors and actuators are added at the outputs and inputs of the plant in order to collect all variations in the plant model in the ' $A$ ' matrix of the plant $P$. The state-space realization of $\mathrm{P}$ can then be written as:

$$
P=\left[\begin{array}{cc}
A(V) & B \\
C & 0
\end{array}\right]
$$

All elements in $\mathrm{A}(\mathrm{V})$ that appear as functions of $\mathrm{V}$ are then considered as "structured" uncertainties and enter the uncertainty block $\Delta_{\mathrm{s}}$. Thus, $\mathrm{A}(\mathrm{V})$ in Eq. (3) becomes:

$A(V)=A_{0}+A_{1} V=A_{0}+47 A_{1}+\left[\begin{array}{cccccccccc}0 & 0 & 0 & 0 & 0 & 0 & 0 & 0 & 0 & 0 \\ 0 & 0 & 0 & 0 & 0 & 0 & 0 & 0 & 0 & 0 \\ 0 & 0 & 0 & 0 & 0 & 0 & 0 & 0 & 0 & 0 \\ 0 & 0 & 0 & 0 & 0 & 0 & 0 & 0 & 0 & 0 \\ 0 & 0 & 0 & 0 & 0 & 0 & 0 & c_{58} \delta_{1} & 0 & 0 \\ 0 & 0 & 0 & 0 & 0 & 0 & c_{67} \delta_{2} & c_{68} \delta_{3} & c_{68} \delta_{4} & 0 \\ 0 & 0 & 0 & 0 & 0 & 0 & 0 & 0 & 0 & 0 \\ 0 & 0 & 0 & 0 & c_{85} \delta_{5} & 0 & 0 & 0 & c_{89} \delta_{6} & 0 \\ 0 & 0 & 0 & 0 & 0 & 0 & 0 & 0 & 0 & 0 \\ 0 & 0 & 0 & 0 & 0 & 0 & 0 & 0 & 0 & 0\end{array}\right]$

where $\mathrm{c}_{\mathrm{ij}}$ are the normalization factors so that the condition $\left|\delta_{i}\right| \leq 1, i=1 . .6$ is satisfied. It is to be noted that a single independent parameter, namely velocity is now replaced with 6 uncertain parameters. The unstructured input multiplicative uncertainty $\Delta_{1}$ helps in incorporating uncertainties that arise due to the fact that many of the elements in $\mathrm{A}(\mathrm{V})$ are the average values of the corresponding elements over the entire flight range. The unstructured uncertainty weighting function $\mathrm{W}$ is included to offset the uncertainties associated with the approximation of state-space elements. $M$ is the matching model which the closed loop system is expected to follow. $\mathrm{W}_{1}$ and $\mathrm{W}_{2}$ are weightings on the error and control effort, respectively. $\mathrm{W}_{2}$ also serves to increase the robustness of the closed loop system to additive uncertainty. $\mathrm{M}$ is chosen to be of diagonal form to achieve decoupling between height and total speed responses:

$$
M=\left[\begin{array}{cc}
0 & 0 \\
\frac{100}{s^{2}+20 s+100} & 0 \\
0 & \frac{100}{s^{2}+20 s+100}
\end{array}\right]
$$

A damping of 1 and frequency of $10 \mathrm{rad} / \mathrm{sec}$ are chosen for the matching model. $P(V)$ is the plant model which varies linearly with trim velocity $V . \mathrm{w}_{\mathrm{g}}$ is the vertical gust obtained as the output of the Dryden model (the input to which is white noise). $d$ is the output disturbance. $K$ is then the $\mu$-synthesis controller to satisfy the performance specification $\left\|T_{z w}\right\|_{\infty}<1$, where $T_{z w}$ is the closed loop transfer function from $w=\left[\begin{array}{c}r \\ d \\ w_{g}\end{array}\right]$ to the performance outputs $z=\left[\begin{array}{c}z_{1} \\ z_{2}\end{array}\right]$ along all variations of $V$. In this design, we choose

$$
W_{1}=\left[\begin{array}{ccc}
\frac{(100 s+1)}{\left(s^{2}+20 s+1\right)} & 0 & 0 \\
0 & \frac{0.07143(\mathrm{~s}+1000)}{(\mathrm{s}+15)} & 0 \\
0 & 0 & \frac{0.01428(\mathrm{~s}+1000)}{(\mathrm{s}+15)}
\end{array}\right] W_{2}=\left[\begin{array}{cc}
\frac{(0.4 s+2)}{(s+80)} & 0 \\
0 & \frac{(0.4 s+2)}{(s+80)}
\end{array}\right]
$$

The interconnection in Fig. 4 can be redrawn as in Fig. 5, where $\mathrm{G}$ is the generalized plant and $\Delta=\operatorname{diag}\left(\Delta_{a}, \delta_{1}, . . \delta_{6}\right)$.

The closed loop system in Fig. 5 is said to be stable and satisfies robust performance if and only if $\left\|F_{u}\left(F_{l}(G, K), \Delta\right)\right\|_{\infty}<1$, for all $\|\Delta\|_{\infty} \leq 1$. The $\mu$ synthesis problem is then to find a controller $\mathrm{K}$ such that the robust performance condition is satisfied. The advantage of using the LPV model is that a single controller that satisfies performance specifications can be designed. The controller $\mathrm{K}$ is designed using the Robust Control Toolbox v3.0 in MATLAB $^{\circledR}$ v7.0.

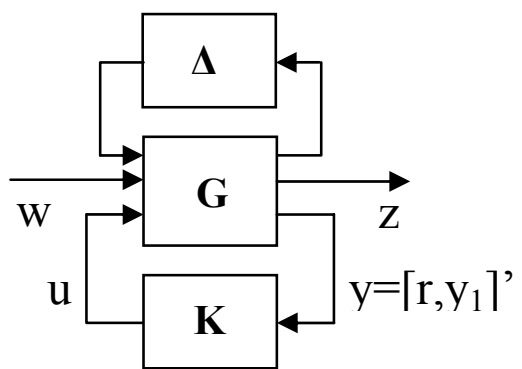

Fig. 5. Representation of the Uncertain Plant under Feedback

Fig. 6 shows the height, total velocity and pitch rate responses to unit step demand at the height reference command channel at speeds of $22-72 \mathrm{~m} / \mathrm{sec}$ at intervals of $5 \mathrm{~m} / \mathrm{sec}$ for the actual closed loop system. It is noted that a single controller is used for achieving height tracking. For purposes of comparison, the open loop responses of the plant at the trim speeds of 22, 37, 52 and $72 \mathrm{~m} / \mathrm{sec}$ are shown in Fig. 7. As can be seen, the closed loop system is well damped and the responses are fast.

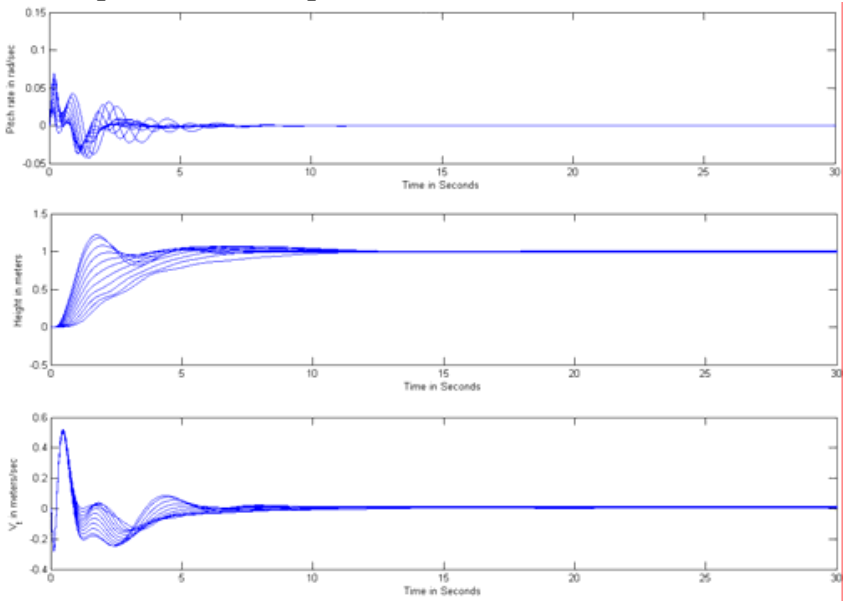

Fig. 6. Step Response of the Closed Loop System at Trim Speeds of $22-$ $72 \mathrm{~m} / \mathrm{sec}$ 

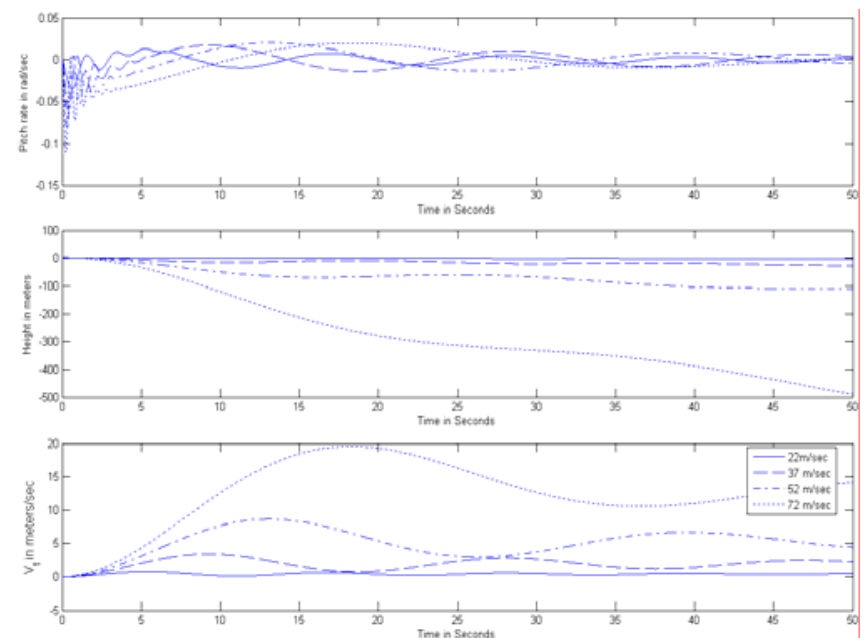

Fig. 7. Step Response of the Open Loop System at Trim Speeds of 22, 37,52 and $72 \mathrm{~m} / \mathrm{sec}$

Fig. 8 shows the $\mu$ bounds for robust stability and Fig. 9 shows the $\mu$ bounds for robust performance of the closed loop system. The closed loop system is robustly stable since the $\mu$ bounds in Fig. 8 are below 1, while the maximum value of the upper $\mu$ bound for robust performance (Fig. 9) is slightly above 1 . The reasonably low value of the upper $\mu$ bound is reflective of the fact that $\mu$ synthesis can be successfully applied to the design of robust controllers for LPV models. However, the design of a single controller could lead to conservativeness in the sense that satisfaction of robust stability over the entire parameter trajectory results in a lowering performance below that which could be achieved if the values of the varying parameter were taken into account in the control action. The $\mu$ framework can still be used for the design of a linearly scheduled controller as will be shown in the next section.

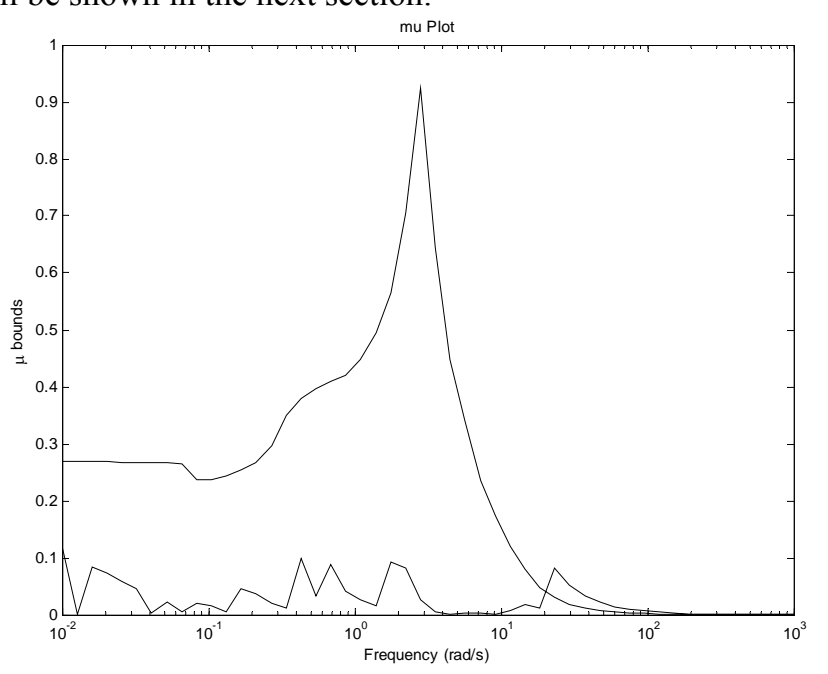

Fig. 8. $\mu$ Bounds for Robust Stability Test

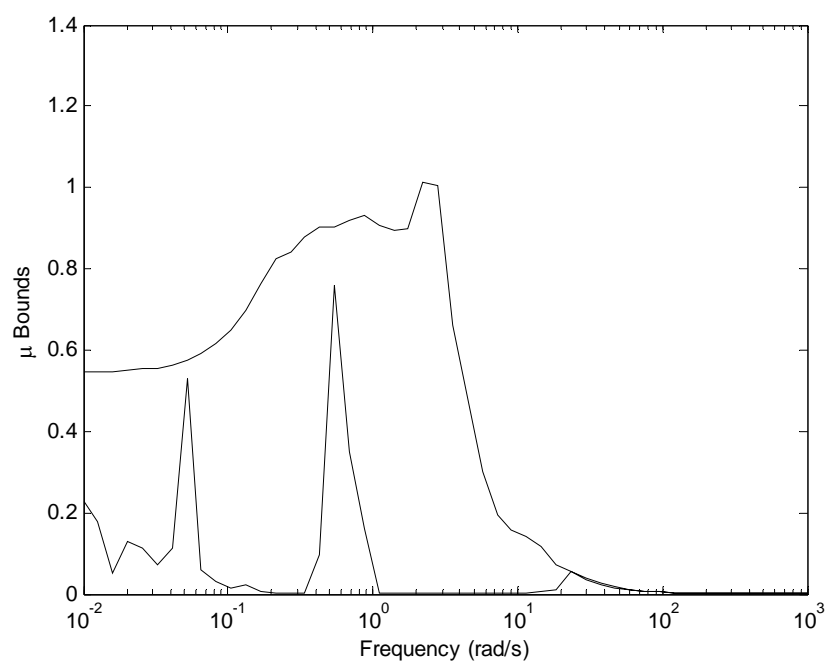

Fig. 9. $\mu$ Bounds for Robust Performance Test

\section{GAIN SCHEDULED CONTROLLER THROUGH $\mu$ SYNTHESIS}

Since the LPV system considered in this paper is polytopic, the LPV controller can also be chosen as a polytopic controller of the form:

$$
K(\theta)=\alpha_{1} K_{1}+\alpha_{2} K_{2}
$$

where $\mathrm{K}_{1}$ and $\mathrm{K}_{2}$ are the controllers designed at the vertices of the velocity polytope, i.e., at 22 and $72 \mathrm{~m} / \mathrm{sec} . \alpha_{1}$ and $\alpha_{2}$ are the solutions of the convex decomposition problem:

$$
\mathrm{V}=\alpha_{1} 22+\alpha_{2} 72
$$

or in the present case, $K(V)=K_{1}\left(1-\alpha_{2}\right)+\alpha_{2} K_{2}$

In fact, this approach is exploited in [6] to reduce the infinite number of constraints imposed by the LMIs arising out of the Quadratic $\mathrm{H}_{\infty}$ performance condition to a finite set of LMIs. However, the complexity of the solution of the LMIs is a disadvantage. In this paper, we use $\mu$ synthesis for the design of LPV polytopic controllers that can be described by Eq. (4). Fig. 10 shows the feedback interconnection for $\mu$ synthesis.

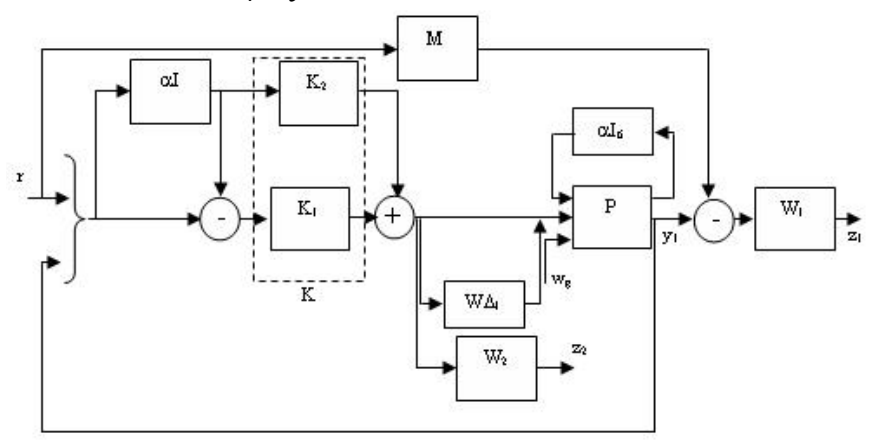

Fig. 10. Closed Loop Interconnection of Gain Scheduled Controller Note that the uncertain parameter $\Delta_{\mathrm{s}}$ in Fig. 4 is now replaced with $\alpha \mathrm{I}_{6}$, where $\alpha=2 \alpha_{2}-1$. As $\mathrm{V}$ varies from 22 to $72 \mathrm{~m} / \mathrm{sec}, \alpha$ varies from -1 to 1 . The controller $\mathrm{K}$ enclosed by the dashed box in Fig. 10 now has 10 inputs corresponding to the 5 inputs of $K_{1}$ and 5 inputs of 
controller $\mathrm{K}_{2}$. The feedback system in Fig. 10 can be recast in the form of Fig. 5 where $\Delta=\operatorname{diag}\left(\alpha I_{11}, \Delta_{1}\right)$. Thus, in effect the scheduling parameter $\alpha$ has been 'collected' into the uncertainties that affect the closed loop system and any variation in the plant model also results in variation in the controller. Controller $\mathrm{K}$ obtained through $\mu$ synthesis yields two constituent controllers, namely $\mathrm{K}_{1}$ obtained by making $\alpha=-1$ and $\mathrm{K}_{2}$ obtained by making $\alpha=1$. Fig. 11 and Fig. 12 show the $\mu$ bounds of the closed loop system in Fig. 10 for robust stability and performance respectively. As can be seen the synthesis of the gain scheduled controller results in better performance with the maximum value of the $\mu$ upper bound less than 1 (0.984). The closed loop responses of the gain-scheduled controller are shown in Fig. 13.

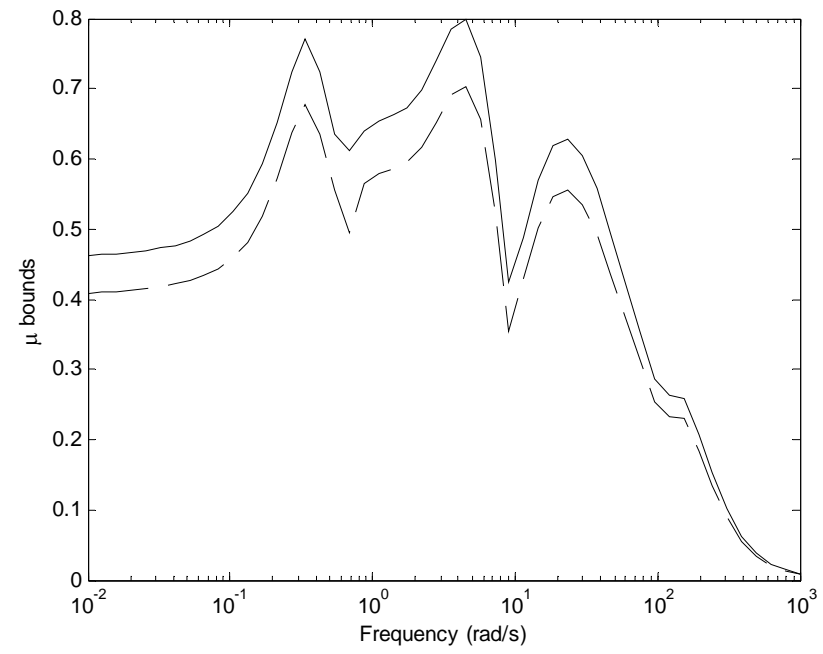

Fig. 11. $\mu$ Bounds for Robust Stability with Gain-Scheduled Controller

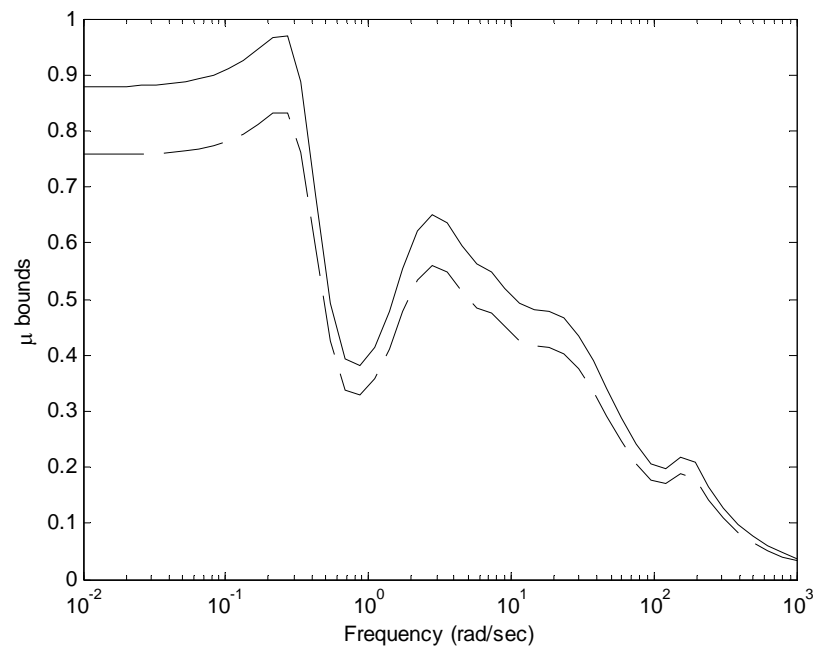

Fig. 12. $\mu$ Bounds for Robust Performance with Gain-Scheduling Controller
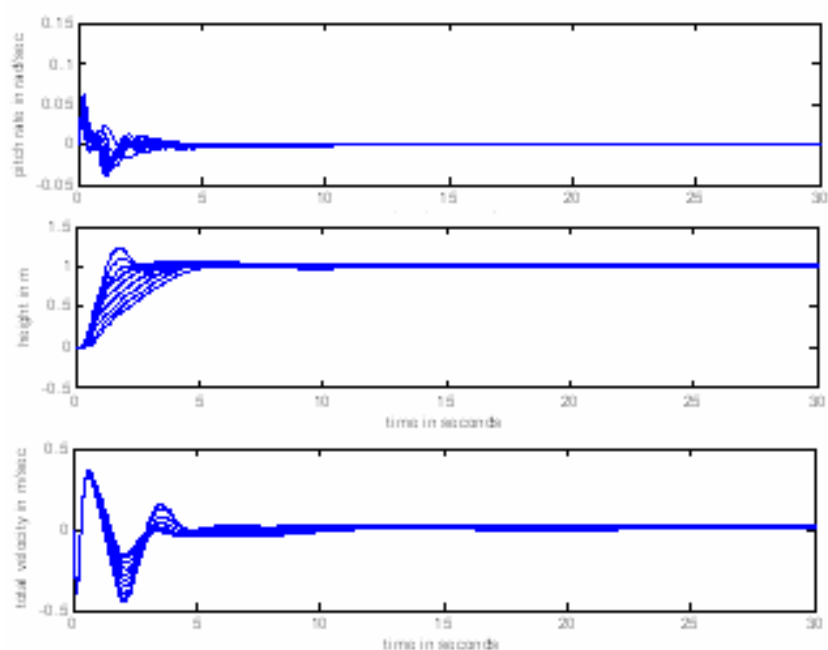

Fig. 13. Closed Loop Responses with Gain-scheduling Controller

Again the closed loop responses are fast and welldamped. However, a more important observation follows from Figs. 6 and 13 with respect to the level of decoupling between the height and total velocity responses. One of the important requirements of the controller is that when the aircraft gains height, there should be minimum variation in total velocity. In fact, this is the reason for the choice of diagonal nature of the matching model. The level of decoupling with the single controller in Fig. 6 as measured by the ratio of the maximum total velocity to the maximum height is 0.41 , while the level of decoupling with the gainscheduled controller is only 0.31 . This reduction in decoupling can again be attributed to the fact that different controllers are used for different plant models.

\section{CONCLUSIONS}

A simplified LPV model based on the full state-space model of the longitudinal dynamics of a UAV is proposed in this paper. Two approaches, based on $\mu$ synthesis are used for controller design for the LPV model. The first design, resulting in a single controller for the entire operating region provides robust stability but is quite conservative in terms of performance. In order to overcome this, a gain scheduled controller is synthesized by treating the scheduling of the controller as a part of the $\mu$ synthesis framework. The gain scheduled control scheme, due to its inherent flexibility, is shown to provide both robust stability and robust performance.

\section{ACKNOWLEDGMENT}

This work is part of a large multi-university project, which is developing new technologies for future UAVs. The authors are grateful to EPSRC and BAE systems for financial support. 


\section{REFERENCES}

[1] J. S. Shamma, and M. Athans, Guaranteed properties of gainscheduled control for linear parameter-varying plants, Automatica, Vol. 27, 1991, pp. $101-107$.

[2] A. Packard, Gain-scheduling via linear fractional transformations, System and Control Letters, Vol. 22, 1994, pp. 79 - 92.

[3] P. Apkarian and P. Gahinet, A convex characterisation of gainscheduling $\mathrm{H}_{\infty}$ congtrollers, IEEE Transactions on Automatic Control, Vol. 40, 1995, pp. $853-864$

[4] A. Packard and G. Becker, Quadratic stabilization of parametrically dependent linear systems using parametrically-dependent linear, dynamic feedback, Advances in robust and nonlinear control systems, 1992. pp. 29-36.

[5] G. Becker, A. Packard, D. Philbrick and G. Balas, Control of parametrically-dependent linear systems: a single quadratic Lyapunov approach, in Proc. American Control Conference, San Fransisco, CA, 1993, pp. $2795-2799$.

[6] P. Apkarian, P. Gahinet and G. Becker, Self-scheduled $\mathrm{H}_{\infty}$ control of linear oarameter-varying systems: a design example, Automatica, Vol. 31,1995 , pp. $1251-1261$.

[7] F. Wang and V. Balakrishnan, Improved stability analysis and gainscheduled controller synthesis for parameter-dependent systems, IEEE Trans on Automatic Control, Vol. 47, 2002, pp 720 - 734.

[8] F. Wu and K. Dong, Gain-scheduled control of LFT systems using parameter-dependent Lyapunov functions, Automatica, Vol. 42, 2006, pp. $39-50$.

[9] G. Balas, I. Fiahlo, A. Packard, J. Refrow and C. Mullaney, On the design of the LPV controllers for the F-14 aircraft lateral-directional axis during powered approach, in Proc. American Control Conference, 1997, pp. 123 - 127.

[10] A. Marocs and G. Balas, Development of linear-parameter varying models for aircraft, Journal of Guidance, Control and Dynamics, Vol. 27,2004, pp. $218-228$. 